\title{
Variation in PCR Efficiencies between Quantification Standards and Clinical Specimens using Different Real-Time Quantitative PCR Interpretation Methods
}

\author{
Byung Ryul Jeon \\ Department of Laboratory Medicine and Genetics, \\ Soonchunhyang University Bucheon Hospital, \\ Soonchunhyang University College of Medicine, \\ Bucheon, Korea
}

\begin{abstract}
The analysis of the $\mathrm{Ct}$ and standard curve produced by real-time polymerase chain reaction (PCR) is a well-established method for the quantification of nucleic acids. However, this method assumes that the PCR efficiency between the unknown specimen and standard is equal, resulting in the possibility of significant inaccuracies due to the presence of inhibitory agents in the unknown specimen. Although numerous methods have been proposed to correct this issue, the understanding of the differences in PCR efficiencies in clinical samples is limited. In this study, 1185 cytomegalovirus (CMV) DNA real-time PCR test results from 106 batches were analyzed. The PCR efficiencies were calculated using the cpD2, maxE, Cy0, maxRatio and window-of-linearity (WoL) methods. The concentrations were calculated using the $\mathrm{cpD} 2$, $\mathrm{Cy0}$, maxRatio, WoL, and take off point (TOP) methods. The coefficient of variation $(\mathrm{CV})$ in the efficiency of the quantification standards was less than $5 \%$ in all methods. Positive samples with high quantification values demonstrated lower PCR efficiency compared to the quantification standards. This suggests possible inaccuracies in quantification using quantification standards in clinical samples.
\end{abstract}

Keywords-PCR Efficiency, Quantitation Algorithm, Realtime PCR, Clinical specimen

\section{INTRODUCTION}

EVERAL methods have been developed for nucleic acid $\mathcal{S}_{\text {quantification. Although recently developed methods such }}$ as next-generation sequencing and droplet digital polymerase chain reaction (PCR) are powerful, they are limited due to the requirement for expensive machinery. Thus, traditional real-time quantitative PCR (qPCR) is widely used in most clinical laboratories[1, 2].

For quantification, most laboratories use the "fit point" method, in which a fixed fluorescence threshold is chosen and the intersection of the fluorescence value of the sample and the threshold yields a quantification cycle $(\mathrm{Cq})$ value[3-5]. The threshold is placed arbitrarily within the early exponential phase of the reaction, where PCR efficiency (E) is presumed to be constant, and assumes equal PCR efficiency between the samples. PCR efficiency is defined as the fold change in the amount of amplicons after each cycle of amplification. However, it is generally acknowledged that the assumption of constant PCR efficiency may be frequently incorrect, and also that small differences in reaction efficiency can lead to considerable quantification errors[2, 6, 7].

Quantification by real-time PCR can be performed using either absolute or relative methods. Relative quantification measures the relative content of a target sequence in a sample compared to that in a reference sample, while the ratio of the target is normalized to the ratio of an endogenous control. Thus, relative quantification is doubly relative and therefore acknowledged to be susceptible to differences in PCR efficiency between samples[1, 3-6].

The absolute method uses known standards and compares the target specimen's Ct value with that of the standard specimen, resulting in the calculation of the exact number of target DNA molecules. The reliability of the absolute method depends on the amplification efficiencies of the target and standard curve[1, 3, 8-10]. However, the differences in amplification efficiency between quantification standards and clinical test samples are not well studied.

In this study, the amplification efficiencies of quantification standards and clinical test samples were analyzed.

\section{II.MATERIALS AND METHODS}

A total 1185 cytomegalovirus (CMV) DNA real-time PCR test results from 106 batches were used in this analysis. One batch consisted of four quantification standards equivalent to 10,000 (QS1), 1,000 (QS2), 100 (QS3), and 10 (QS4) copies/mL, one negative control, and test samples. Tests were performed using the Rotor-Gene Q real-time PCR machine (QIAGEN, Hilden, Germany) and the Artus CMV RG PCR Kit (QIAGEN, Hilden, Germany). Data analyses were performed using the $\mathrm{R}$ statistical environment combined with the qpcR 
package (http://www.dr-spiess.de/qpcR.html). PCR efficiency was calculated using the cpD2, maxE, Cy0, maxRatio and window-of-linearity (WoL) methods. The efficiency was calculated using an efficiency curve, which uses a fitted curve in all methods except for the maxRatio method. However, each of these methods uses different points of the efficiency curve to calculate efficiency. The cpD2 method uses the maximum of the second derivative curve, the maxE method uses the maximum of the efficiency curve, the WoL method uses the exponential region of the curve, and the Cy0 method uses the intersection of a tangent of the first derivative maximum. In the maxRatio method, the maximum efficiency is calculated from the cubic spline interpolated raw fluorescence values and therefore is not calculated from a sigmoidal fit. For the quantification, prediction was performed using the fitted model with the threshold cycle of the quantification standards calculated using the cpD2, Cy0, maxRatio, WoL, and take off point (TOP) methods. The default 14 model in qpcR was used for the sigmoidal fit and default parameters were used for the analyses. Mean results were compared using a one-way ANOVA followed by Tukey's Honestly Significant Difference (HSD) post-hoc tests. $P$ values $<0.05$ were considered statistically significant.

\section{RESULTS}

The PCR efficiency of each of the 106 batches containing four quantification standards and one negative control standard and 499 total sample results, which consisted of 23 positive samples that were calculated to contain over 100 copies/mL the target DNA sequence in all five methods (high copy samples) and 476 positive samples that were calculated to contain less than 100 copies/mL in at least one method(low copy samples), were analyzed using the cpD2, maxE, Cy0, maxRatio, and WoL methods (Table 1). Efficiency was significantly different among methods $(P<0.05)$ except between maxRatio and maxE methods. All five methods had coefficient of variation (CV) values of less than $5 \%$ in the quantification standards. The PCR efficiency measured using the Cy0 method had the lowest value and the CV of the efficiency measured using the Cy0 method was the lowest value among all levels of the quantification standard. However, the Cy0 method had the highest CV in PCR efficiency of the negative control standard. In the high copy samples, the mean PCR efficiencies calculated using the cpD2, maxE, Cy0, maxRatio, and WoL methods were 1.03, 1.04, $1.01,1.05$, and 1.05 , respectively. In the low copy samples, the mean PCR efficiencies measured using the cpD2, maxE, Cy0, maxRatio, and WoL methods were 1.09, 1.12, 1.03, 1.10, and 1.15, respectively. The mean PCR efficiencies of the positive samples were all less than those of the quantification standard. The CV values for the high copy samples were $7.47 \%, 6.98 \%$, $5.90 \%$, 5.48\%, and $2.83 \%$ using the WoL, maxRatio, maxE, cpD2, and Cy0 methods, respectively. The CV values for the low copy samples were $10.18 \%, 8.20 \%, 8.09 \%, 7.06 \%$, and $6.28 \%$ using the maxE, maxRatio, $\mathrm{cpD} 2$, WoL, and Cy0 methods, respectively. Although the $\mathrm{CV}$ values of the PCR efficiencies of the high copy samples measured using the Cy0 methods were less than those of the quantification standards, all other methods demonstrated increased CV values in the high copy samples. In addition, the CV values of the low copy samples were higher than those calculated for the high copy samples using all methods. The concentrations of the quantification standards were calculated using the fitted model from the threshold cycle of the standards. The calculated concentrations were significantly different between WoL methods TOP methods. The CV values calculated using the cpD2 and maxRatio methods were less than $10 \%$ and those calculated using the Cy0 method were less than $15 \%$. The WOL and TOP methods had CV values of over $100 \%$ in some quantification standard samples (Table 2).

Table I. Efficiencies of standard and positive samples.

\begin{tabular}{llccccc}
\hline & & cpD2 & maxE & Cy0 & $\begin{array}{c}\text { max } \\
\text { Ratio }\end{array}$ & WoL \\
\hline QS1 & Mean & 1.20 & 1.21 & 1.11 & 1.25 & 1.26 \\
$(10,000$ & SD & 0.05 & 0.06 & 0.04 & 0.05 & 0.05 \\
copies/mL) & CV (\%) & 4.52 & 4.55 & 3.63 & 4.03 & 4.34 \\
\hline QS2 & Mean & 1.20 & 1.21 & 1.10 & 1.24 & 1.25 \\
$(1,000$ & SD & 0.05 & 0.05 & 0.04 & 0.05 & 0.05 \\
copies/mL) & CV (\%) & 4.28 & 4.26 & 3.55 & 4.09 & 4.37 \\
\hline QS3 & Mean & 1.19 & 1.21 & 1.09 & 1.23 & 1.24 \\
$(100$ & SD & 0.05 & 0.05 & 0.04 & 0.05 & 0.05 \\
copies/mL) & CV (\%) & 4.03 & 3.97 & 3.36 & 3.90 & 4.17 \\
\hline QS4 & Mean & 1.19 & 1.21 & 1.08 & 1.21 & 1.24 \\
$(10$ & SD & 0.05 & 0.05 & 0.03 & 0.05 & 0.05 \\
copies/mL) & CV (\%) & 3.91 & 3.84 & 3.22 & 3.72 & 3.86 \\
\hline & Mean & 1.00 & 1.00 & 1.01 & 1.01 & 1.01 \\
NTC & SD & 0.00 & 0.00 & 0.02 & 0.01 & 0.00 \\
& CV (\%) & 0.11 & 0.13 & 2.14 & 0.55 & 0.38 \\
\hline High Copy & Mean & 1.03 & 1.04 & 1.01 & 1.05 & 1.05 \\
Samples & SD & 0.06 & 0.06 & 0.03 & 0.07 & 0.08 \\
(>100 & CV (\%) & & & & 6.98 & 7.47 \\
copies/mL, & & 5.48 & 5.90 & 2.83 & & \\
N=23) & & & & & & 1.15 \\
\hline Low Copy & Mean & 1.09 & 1.12 & 1.03 & 1.10 & 1.15 \\
Samples & SD & 0.09 & 0.11 & 0.06 & 0.09 & 0.08 \\
$(<=100$ & CV(\%) & & & & 8.20 & 7.06 \\
copies/mL, & & 8.09 & 10.18 & 6.28 & & \\
N=476) & & & & & & \\
\hline
\end{tabular}


Table II. Calculated results of quantification standards.

\begin{tabular}{llrrrrr}
\hline & & cpD2 & Cy0 & $\begin{array}{r}\text { maxRatio } \\
\text { (adjusted) }\end{array}$ & WoL & TOP \\
\hline st1 & Mean & 10361.96 & 10329.58 & 9912.55 & 13916.25 & 7451.81 \\
$(10,000$ & SD & 580.42 & 1034.93 & 570.70 & 3708.83 & 5095.38 \\
copies/mL) & CV (\%) & 5.60 & 10.02 & 5.76 & 26.65 & 68.38 \\
\hline st2 & Mean & 950.44 & 951.86 & 998.34 & 374.21 & 1263.36 \\
$(1,000$ & SD & 70.48 & 127.04 & 77.67 & 362.07 & 1336.43 \\
copies/mL) & CV (\%) & 7.42 & 13.35 & 7.78 & 96.76 & 105.78 \\
\hline st3 & Mean & 99.79 & 101.39 & 103.54 & 95.85 & 241.35 \\
$(100$ & SD & 7.66 & 13.24 & 10.18 & 109.76 & 348.55 \\
copies/mL) & CV (\%) & 7.68 & 13.06 & 9.83 & 114.51 & 144.41 \\
\hline st4 & Mean & 10.27 & 10.36 & 9.87 & 46.96 & 47.37 \\
$(10$ copies/mL) & SD & 0.70 & 1.54 & 0.66 & 62.65 & 90.13 \\
& CV (\%) & 6.84 & 14.90 & 6.70 & 133.40 & 190.25 \\
\hline
\end{tabular}

\section{DISCUSSION}

Real-time PCR quantification methods are based on the monitoring of the increasing fluorescence intensity derived from a double-strand-specific dye such as SYBR Green I, which increases after each PCR cycle. PCR efficiency is defined as the fold change in the amount of amplicons after each cycle of amplification[2, 5].

For absolute quantification by real-time PCR, the standard curve method is widely used, which is based on the cycle-threshold values of either an input series of known RNA concentrations (in reverse-transcription qPCR) or a dilution series of a reference cDNA[2].

Cycle-threshold is defined as the fractional cycle number in the log-linear region of the PCR amplification, in which the reaction produces fixed amounts of amplicon DNA. The fit point method draws an arbitrary line parallel to the $\mathrm{x}$-axis of the real-time fluorescence intensity curve $(\mathrm{Ct})$ in an early phase of detectable amplification. The second derivative method calculates the fractional cycle in which the second derivative of the real-time fluorescence intensity curve reaches the maximum value (the crossing point, $\mathrm{Cp}$ ). These methods assume that the amplification efficiencies of the standard and the testing samples are equal[ $[7,11]$.

However, this assumption is not always true. Inefficient PCR amplification may result from an insufficient quantity of DNA or the presence of inhibitors such as cetrimonium bromide (CTAB), ethylenediaminetetraacetic acid (EDTA), ethanol, fat, isopropanol, phenol, polysaccharides, protein, sodium dodecyl sulfate (SDS), sodium acetate, or sodium chloride in the DNA preparation. These inhibitors can be included in the specimen or added during the testing process[8]. In test samples, the possible presence of inhibitors complicates data analyses. In addition to the presence of inhibitors, PCR efficiency can be influenced by the applied baseline correction method[12].

To overcome these limitations, many attempts have been made using individual reaction efficiencies to compensate for such effects, to remove reactions with aberrant PCR efficiencies from the analyses, or to develop algorithms that are less influenced by differences in PCR efficiency[1, 2, 5-7, 9-15].
The Cy0 method is based on a modified standard curve and attempts to minimize the effects of varying PCR efficiencies by relying on the inflection point position and the slope of the fluorescence curve, which do not require the assumption of uniform reaction efficiency between the standards and unknown samples [6].

The TOP method calculates the first significant cycle of the exponential region by statistical delimitation from a single reaction setup [4].

The maxRatio method can measure a consistent point within or very near to the exponential region of the PCR fluorescence signal without requiring user intervention, even in the presence of systematic and nonsystematic errors in amplification signals [11].

The WoL method uses an iterative algorithm to search for the highest R2 value and a slope close to the maximum slope to provide an assumption-free method to calculate starting concentrations of mRNAs and PCR efficiencies for each sample [7].

In this study, we investigated the differences in the PCR efficiencies between the quantification standard included in the commercial test kit and clinical test samples and found that, using all of the above methods, the PCR efficiencies of the test samples were lower than those of the quantification standards. Clinical laboratory report test results with the assumption that the efficiency between the quantitation standard of the commercial test kit and the clinical test sample using the same test kit would be the same. This suggests the possible source of the inaccuracy of the quantification of nucleic acids when using commercial test kits.

The CV values of the determined concentrations demonstrated significant differences between quantification methods. As these methods were dependent on the fitted model that was used, the selection and adjustment of the model that is applied is critical to the performance of the quantification. However, this study was limited in that the analysis methods were applied using the default parameters and models. For a more accurate comparison of the performance of these methods, further study should include the optimization of the models that are applied. 


\section{REFERENCES}

[1] Chen P and Huang X. Comparison of analytic methods for quantitative real-time polymerase chain reaction data. J Comput Biol 2015; 22: 988-996.

[2] Ji W, Li J and Liu J. A method for measuring gene copy number in biological samples without using control samples of known copies. J Mol Diagn 2012; 14: 280-285

[3] Tellinghuisen $\mathrm{J}$ and Spiess AN. Bias and imprecision in analysis of real-time quantitative polymerase chain reaction data. Anal Chem 2015; 87: 8925-8931.

[4] Tichopad A, Dilger M, Schwarz G and Pfaffl MW. Standardized determination of real-time PCR efficiency from a single reaction set-up. Nucleic Acids Res 2003; 31: e122.

[5] Cikos S, Bukovska A and Koppel J. Relative quantification of mRNA: comparison of methods currently used for real-time PCR data analysis. BMC Mol Biol 2007; 8: 113.

[6] Guescini M, Sisti D, Rocchi MB, Stocchi L and Stocchi V. A new real-time PCR method to overcome significant quantitative inaccuracy due to slight amplification inhibition. BMC Bioinformatics 2008; 9: 326.

[7] Ramakers C, Ruijter JM, Deprez RH and Moorman AF. Assumption-free analysis of quantitative real-time polymerase chain reaction (PCR) data. Neurosci Lett 2003; 339: 62-66.

[8] Demeke T and Jenkins GR. Influence of DNA extraction methods, PCR inhibitors and quantification methods on real-time PCR assay of biotechnology-derived traits. Anal Bioanal Chem 2010; 396: 1977-1990.

[9] Spiess AN, Feig C and Ritz C. Highly accurate sigmoidal fitting of real-time PCR data by introducing a parameter for asymmetry. BMC Bioinformatics 2008; 9: 221.

[10]Tellinghuisen J and Spiess AN. qPCR data analysis: Better results through iconoclasm. Biomol Detect Quantif 2019; 17: 100084.

[11]Shain EB and Clemens JM. A new method for robust quantitative and qualitative analysis of real-time PCR. Nucleic Acids Res 2008; 36: e91.

[12]Ruijter JM, Ramakers C, Hoogaars WM, Karlen Y, Bakker O, van den Hoff MJ and Moorman AF. Amplification efficiency: linking baseline and bias in the analysis of quantitative PCR data. Nucleic Acids Res 2009; 37: e45.

[13]Kolacsek O, Pergel E, Varga N, Apati A and Orban TI. Ct shift: A novel and accurate real-time PCR quantification model for direct comparison of different nucleic acid sequences and its application for transposon quantifications. Gene 2017; 598: 43-49.

[14]Rutledge RG and Stewart D. A kinetic-based sigmoidal model for the polymerase chain reaction and its application to high-capacity absolute quantitative real-time PCR. BMC Biotechnol 2008; 8: 47.

[15]Tellinghuisen J and Spiess AN. Absolute copy number from the statistics of the quantification cycle in replicate quantitative polymerase chain reaction experiments. Anal Chem 2015; 87: 1889-1895.

\section{Creative Commons Attribution License 4.0 (Attribution 4.0 International, CC BY 4.0)}

This article is published under the terms of the Creative Commons Attribution License 4.0 https://creativecommons.org/licenses/by/4.0/deed.en_US 